\title{
Alpha-Methaylcayl-CoA racemase expression is associated with early lesions of prostate cancer.
}

Hanna M. Romanska, Ryszard Maranda ${ }^{2}$, Piotr Marks ${ }^{3}$, Boguslaw Olborski ${ }^{4}$, Gordon W. H. Stamp, Paul D. Abel ${ }^{1}$, El-Nasir Lalani.

Departments of Histopathology and ${ }^{1}$ Surgery, Imperial College, London, U.K, ${ }^{2}$ Department of Urology, Hospital in Tuszyn, Poland and Departments of ${ }^{3}$ Urology and ${ }^{4}$ Pathomorphology, John Paul II Hospital in Belchatow, Poland.

Corresponding author: Prof. El-Nasir Lalani, Department of Histopathology, Hammersmith Campus, Du Cane Road, London W12 ONN. Tel: 020-8383-2430; Fax: 020-8383-2431.

\section{Introduction.}

Prostate cancer (PC) accounts for almost $10 \%$ of all malignancies in men with half a million new cases occurring each year. Serum level of PSA is currently the most commonly used biochemical marker for both diagnosis and monitoring of cancer progression and response to therapy, but its specificity and precise place in early detection and staging are still questionable. There is, therefore, a pressing need for more accurate screening methods and identification of new genes abnormally expressed in early malignant lesions. Emerging evidence seems to suggest that $\alpha$-methylacyl-CoA racemase (AMACR), a cytoplasmic protein involved in peroxisomal $\beta$-oxidation of branched-chain fatty acids may fulfil these requirements.

\section{Aim}

The aim of our study was to assess usefulness of immunohistochemistry for AMACR in PC detection.

\section{Material and Methods.}

Archival surgical specimens of: 1) PC (radical prostatectomy $n=5$; Gleason I/II and II/III; needle biopsy $n=3)$, 2) BPH (n=4), 3) transitional cell carcinoma (TCC) of the bladder (grade II and III; $n=13$ ), 4) yolk sac tumour of the testis $(n=2)$ and 5 ) hydrocoele of the testis $(n=1)$ fixed in $10 \%$ formalin were processed for immunohistochemistry. Paraffin sections were microwaved in citrate buffer $(\mathrm{pH}=6.0)$ and immunostaining was carried out using $A B C$-peroxidase complex method.

\section{Results.}

AMACR was found to be expressed in PCs but not in the normal glands within the same specimen or in BPH samples. Only weak heterogeneous expression was detectable in TCCs of the bladder. There was no differential expression in benign and malignant testicular samples examined.

\section{Conclusion.}

In this pilot study we have demonstrated de novo AMACR expression in PCs. Further studies evaluating AMACR as a specific prognostic marker in PC are being undertaken. 